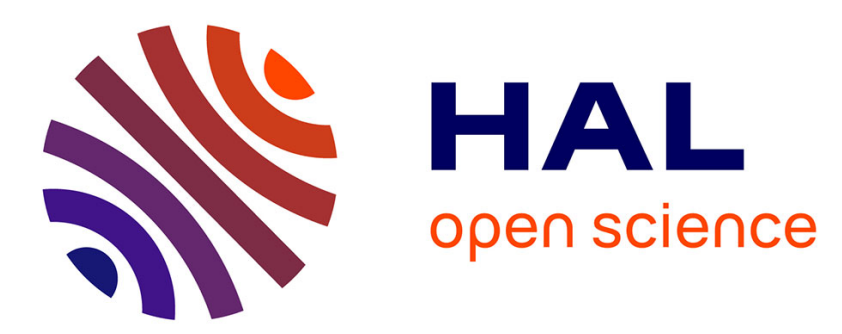

\title{
Transport dans la couche d'inversion d'un transistor MOS. Utilité du formalisme de Kubo-Greenwood
}

G. Ghibaudo

\section{To cite this version:}

G. Ghibaudo. Transport dans la couche d'inversion d'un transistor MOS. Utilité du formalisme de Kubo-Greenwood. Revue de Physique Appliquée, 1986, 21 (2), pp.121-129. 10.1051/rphysap:01986002102012100 . jpa-00245417

HAL Id: jpa-00245417

https://hal.science/jpa-00245417

Submitted on 1 Jan 1986

HAL is a multi-disciplinary open access archive for the deposit and dissemination of scientific research documents, whether they are published or not. The documents may come from teaching and research institutions in France or abroad, or from public or private research centers.
L'archive ouverte pluridisciplinaire $\mathbf{H A L}$, est destinée au dépôt et à la diffusion de documents scientifiques de niveau recherche, publiés ou non, émanant des établissements d'enseignement et de recherche français ou étrangers, des laboratoires publics ou privés. 


\title{
Transport dans la couche d'inversion d'un transistor MOS. Utilité du formalisme de Kubo-Greenwood
}

\author{
G. Ghibaudo \\ Laboratoire de physique des composants à semiconducteurs, \\ ERA-CNRS 659, ENSERG, 23, avenue des Martyrs, 38031 Grenoble Cedex, France
}

(Reçu le 22 février 1984, révisé les 20 novembre 1984 et 30 mai 1985, accepté le 8 novembre 1985)

\begin{abstract}
Résumé. - Une étude du transport électronique dans la couche d'inversion d'un transistor MOS est présentée. L'utilisation du formalisme de Kubo-Greenwood rend possible l'explication du comportement global des coefficients de transport (conductivité, mobilité, ...) du régime d'inversion faible au régime d'inversion forte sur une gamme étendue de température. En particulier, il est montré que des phénomènes physiques découlant de la miniaturisation et/ou d'un fonctionnement à basse température, tels que la quantification de la couche d'inversion ou la localisation des porteurs, sont de première importance en ce qui concerne l'évaluation des coefficients de transport dans la couche d'inversion de T.MOS. La prise en compte de ces phénomènes lors de la modélisation du fonctionnement de T.MOS, est donc fortement souhaitable, même pour des températures proches de l'ambiante.

Abstract. - A study of the electronic transport in MOS transistor inversion layers is presented. The use of the Kubo-Greenwood formalism allow the possibility to explain the global behaviour of the transport coefficients (conductivity, mobility, ...) from the weak inversion regime (non degenerate) to the strong inversion one (degenerate) over a wide range of temperature. In particular, it is shown that physic phenomena arising from miniaturization and/or low temperature working such as the quantification of the inversion layer or the carrier localization, are of first importance for the determination of the transport coefficients of MOST inversion layers. The account of such phenomena into the modelling of MOST working, is thus greatly desirable, even at temperatures close to room ones.
\end{abstract}

\section{Introduction.}

Les études du transport électronique dans les couches d'inversion de transistors MOS (métal-oxyde-semiconducteur) (T.MOS) présentent un double intérêt. Premièrement, elles procurent d'énormes possibilités d'investigations quant aux mécanismes fondamentaux $\mathrm{du}$ transport électronique à l'interface oxyde-semiconducteur grâce à la facilité de découplage entre la température et la concentration des porteurs [1]. D'autre part, ces études sont de première importance en ce qui concerne la modélisation des lois macroscopiques qui régissent le fonctionnement des dispositifs basés sur de tels composants (T.MOS) [2, 3].

En outre, l'amélioration des performances des transistors MOS de la microélectronique actuelle, nécessite soit une miniaturisation des composants (VLSI), soit un fonctionnement du T.MOS à basse température (MOS « cryogénique »).

De ces conditions particulières de fonctionnement résultent des limitations quant aux hypothèses phy- siques qui prévalent à l'heure actuelle lors de la modélisation du transport dans les T.MOS à température ambiante :

- traitement classique de la couche d'inversion du canal,

- emploi de la statistique de Boltzmann,

- absence d'effet de localisation des porteurs...

En effet, il est maintenant établi que les effets de quantification par le champ électrique de "grille " dans la couche d'inversion de T.MOS se manifestent même à la température ambiante et sont d'autant plus importants que la température est basse $[1,4]$.

De plus, la miniaturisation des composants implique une réduction des dimensions du T.MOS et en particulier de l'épaisseur de l'oxyde de grille, et partant un accroissement du champ électrique de surface qui favorise ainsi la dégénérescence statistique des porteurs dans le canal d'inversion entraînant la nonvalidité de la statistique de Boltzmann.

Enfin, les phénomènes de localisation qui provien- 
nent de la présence de fluctuations de potentiel de surface et qui se traduisent par une diminution effective de la mobilité des porteurs, sont accrus et amplifiés lors d'un fonctionnement à basse température du T.MOS $[1,8]$.

L'objectif de cet article n'est pas de présenter une modélisation fine du transport dans la couche d'inversion d'un transistor MOS qui tienne compte de paramètres multiples (tensions de polarisation, température de fonctionnement, conditions technologiques...), mais davantage de proposer une approche du transport qui prenne en compte autant que possible les phénomènes physiques inhérents aux principales limitations invoquées précédemment (quantification de la couche d'inversion, statistique de Boltzmann, désordre). A cette fin, nous utilisons un formalisme du transport généralisé propre aux matériaux désordonnés [5] (formalisme de Kubo-Greenwood), dont l'intérêt de la formulation valable quel que soit l'état de dégénérescence, est d'être basée sur les grandeurs fondamentales relevant du transport dans une bande d'énergie tels que la densité d'états $N(E)$ et la mobilité énergétique $\mu(E)$. Cette formulation nous permettra, moyennant quelques hypothèses simplificatrices quant aux mécanismes de collisions des porteurs, de décrire et d'expliquer dans leur globalité, les variations des coefficients de transport (conductivité, mobilité...) avec la température et/ou la concentration des porteurs dans le canal de la couche d'inversion.

\section{Statistique de la couche d'inversion.}

Dans cette partie nous rappelons les conséquences de la quantification par le champ électrique de "grille» sur la densité d'états effective du gaz quasi bidimensionnel qui se forme au voisinage de l'interface oxydesemiconducteur. Nous donnons une formulation de la concentration de porteurs valable du régime semiconducteur au régime métallique. Enfin, nous indiquons les évolutions du niveau de Fermi et de la longueur d'écran électrostatique avec la température ou la concentration de porteurs.

\subsection{QuANTIFICATION DE LA COUCHE D'INVERSION. -} La quantification de la couche d'inversion résulte de la présence du fort champ électrique perpendiculaire à l'interface oxyde-semiconducteur dû à la polarisation de grille $[1,6]$. La discrétisation de l'énergie dans la direction perpendiculaire à la surface entraîne la formation de sous-bandes bidimensionnelles. Les niveaux énergétiques discrets $E_{i}$ définissant les bords de chaque sous-bande, sont reliés à l'intensité du champ électrique de surface $F_{\mathrm{s}}$ par une relation de la forme $[6,7]$ :

$$
E_{i}=\frac{\left(q \hbar F_{\mathrm{s}}\right)^{2 / 3}}{\left(2 m^{*}\right)^{1 / 3}} A_{i}
$$

où les $A_{i}$ sont les zéros de la fonction d'Airy, $m^{*}$ la masse effective, $\hbar$ la constante de Planck, $q$ la charge absolue de l'électron. Par exemple, la différence d'énergie entre les deux premiers niveaux $E_{1}$ et $E_{2}$ vaut typiquement $80 \mathrm{meV}$ pour un champ de surface modéré de $5 \times 10^{5} \mathrm{~V} / \mathrm{cm}$ (équivalent à $2,5 \mathrm{~V}$ sur $500 \AA$ ). Cela montre que les effets quantiques ne peuvent être négligés même à la température ambiante puisque l'agitation thermique n'atteint que $25 \mathrm{meV}$.

La densité d'électrons $N_{i}$ peuplant la $i$-ème sousbande est donné par $[4,6]$ :

$$
N_{i}=\left(\frac{m^{*}}{\pi \hbar^{2}}\right) k T \log \left(\exp \left(\frac{E_{\mathrm{F}}-E_{i}}{k T}\right)+1\right)
$$

où $k$ est la constante de Boltzmann, $T$ la température absolue et $E_{\mathrm{F}}$ le niveau de Fermi commun à toutes les sous-bandes.

La densité totale d'électrons $N_{\mathrm{s}}$ dans la couche d'inversion provient de la contribution de chaque sous-bande et vaut :

$$
N_{\mathrm{s}}=\sum_{i} N_{i}
$$

En général la charge d'inversion $Q_{i}=-q N_{\mathrm{s}}$ dépend de l'intensité du champ de surface par l'intermédiaire de la relation de Gauss :

$$
F_{\mathrm{s}}=\frac{Q_{i}+Q_{\mathrm{D}}}{\varepsilon}
$$

où est $Q_{\mathrm{D}}$ est la charge de déplétion dans le semiconducteur, $\varepsilon$ la permittivité diélectrique.

L'autoconsistence du système d'équations (1) à (4) permet, en général, de déterminer la position du niveau de Fermi en fonction du champ de surface et de la température.

En pratique, pour des raisons de commodité, on considèrera que seule la sous-bande fondamentale $E_{0}$ est occupée; cela sera d'autant mieux vérifié que la température sera basse. De toute façon, même à la température ambiante, plus de $50 \%$ des porteurs se trouvent déjà dans la sous-bande fondamentale [7], ce qui justifie en partie le choix de cette approximation.

\subsection{Evolution du Niveau de Fermi avec la tempé-} RATURE. - Dans le cas d'une couche d'inversion ne comportant qu'une seule sous-bande, le niveau de Fermi est relié directement à la concentration de porteurs $N_{\mathrm{s}}$ (\# $N_{0}$, densité dans la sous-bande fondamentale) par l'intermédiaire de la relation (2).

L'évolution du niveau de Fermi avec la température est représentée sur la figure 1 pour différentes concentrations d'électrons $N_{\mathrm{s}}$ dans la couche d'inversion. On peut noter que, dès que la température est suffisamment basse, le niveau de Fermi passe au-dessus $\mathrm{du}$ bord de bande et se stabilise à une valeur caractéristique du régime métallique : $E_{\mathrm{F}}=E_{0}+$ $N_{\mathrm{s}}\left(\pi \hbar^{2} / m^{*}\right)$.

A partir de la relation (2), on peut définir la con- 


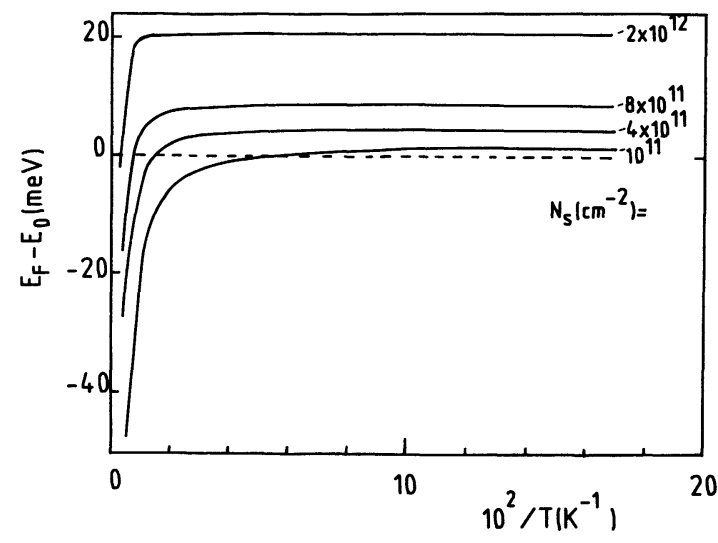

Fig. 1. - Evolution du niveau de Fermi $E_{\mathrm{F}}$ avec l'inverse de la température $10^{2} / T$ pour différentes concentrations de porteurs $N_{\mathrm{s}}$ dans une couche d'inversion ne comportant qu'une seule sous-bande.

[Evolution of the Fermi level $E_{\mathrm{F}}$ with reverse temperature $10^{2} / T$ for different carrier concentrations $N_{\mathrm{s}}$ in an inversion layer having only one subband.]

centration de porteurs critiques $N_{\text {deg }}$ à laquelle apparaît la dégénérescence $\left(E_{\mathrm{F}}=E_{0}\right)$ :

$$
N_{\mathrm{deg}}=\frac{m^{*}}{\pi \hbar^{2}} \log 2 k T
$$

La droite $N_{\text {deg }}$ en fonction de $T$ partage l'espace des concentrations de porteurs et des températures en deux régions. Lorsque la concentration $N_{\mathrm{s}}$ est inférieure à $N_{\text {deg }}$, la couche d'inversion n'est pas dégénérée : la statistique de Boltzmann est applicable. Lorsque la concentration $N_{\mathrm{s}}$ est supérieure à $N_{\text {deg }}$, la couche d'inversion dégénère : la statistique de Boltzmann devenant caduque, la statistique dégénérée prévaut.

Il est à noter que, à $300 \mathrm{~K}, 3 \times 10^{12}$ charges $/ \mathrm{cm}^{2}$ provoquent la dégénérescence de la couche d'inversion (ce qui correspond d'après la relation (4) à un champ $F_{\text {s }} \# 5 \times 10^{5} \mathrm{~V} / \mathrm{cm}$ ), alors qu'à $77 \mathrm{~K}, 7 \times 10^{11}$ charges $/ \mathrm{cm}^{2}$ sont suffisantes pour produire le même effet. Cela met en exergue le fait qu'il serait abusif d'utiliser sans précaution la statistique de Boltzmann dans les modèles de T.MOS fonctionnant à basse température ou sous forte polarisation.

\subsection{EVOLUTION DE LA CONSTANTE D'ÉCRAN ÉLECTRO-} STATIQUE. - Cette longueur caractérise la distance sur laquelle toute perturbation locale du potentiel électrique est atténuée par la charge du gaz bidimensionnel de porteurs libres. Son rôle est particulièrement important en ce qui concerne l'influence des charges fixes situées près de l'interface oxyde-semiconducteur, sur les variations locales du potentiel de surface. Elle intervient également sur la section efficace de diffusion des mécanismes de collisions sur les centres chargés.

Dans l'approximation de Thomas-Fermi et pour un gaz à deux dimensions (2D), l'expression de la longueur d'écran $\lambda_{2 \mathrm{D}}$ pour une seule sous-bande est de la forme [8] :

$$
\lambda_{2 \mathrm{D}}=\frac{\varepsilon}{q^{2}} \frac{\delta E_{\mathrm{F}}}{\delta N_{\mathrm{s}}} .
$$

Dans le cas de plusieurs sous-bandes, l'écrantage total résulte de la contribution de l'écrantage partiel de chaque sous-bande et s'obtient par [8] :

$$
\lambda_{2 \mathrm{D}}=\left(\sum_{i} 1 / \lambda_{i}\right)^{-1}
$$

où les $\lambda_{i}$ sont les longueurs d'écran relatives à la $i$-ème sous-bande et sont donnés par une relation du type de la relation (6) dans laquelle $N_{\mathrm{s}}$ est remplacé par $N_{i}$.

En utilisant les relations (2) et (6) on obtient les variations de la longueur d'écran $\lambda_{2 \mathrm{D}}$ dans l'approximation d'une couche d'inversion à une seule sousbande en fonction de la température $T$ et de la concentration $N_{\mathrm{s}}$ représentées sur la figure 2 . On constate que $\lambda_{2 \mathrm{D}}$ prend une valeur constante minimale, de l'ordre de $3 \AA$, soit lorsque la température est basse, soit lorsque la concentration de porteurs est forte; c'est-à-dire lorsque la statistique dégénérée prédomine. En revanche, à température fixée, $\lambda_{2 D}$ augmente rapidement (en $N_{\mathrm{s}}^{-1}$ ) dès que la concentration devient inférieure à $N_{\text {deg }}(T)$ (cf. Fig. 2), la statistique de Boltzmann devenant effective.

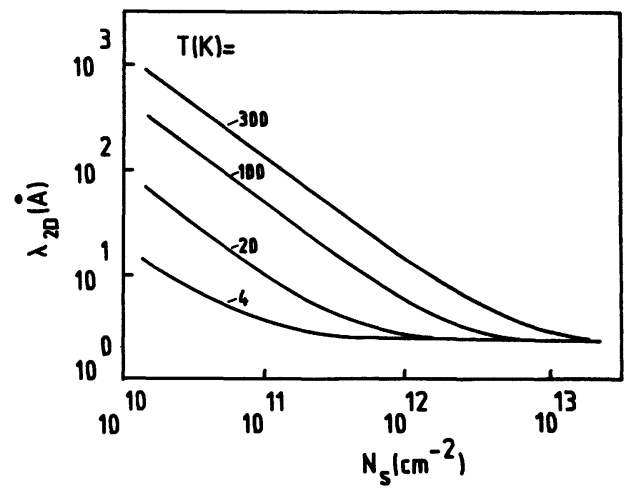

Fig. 2. - Variations de la longueur d'écran $\lambda_{2 \mathrm{D}}$ avec la concentration de porteurs $N_{\mathrm{s}}$ pour différentes températures.

[Variations of the screening length $\lambda_{2 D}$ with carrier concentration $N_{\mathrm{s}}$ for different temperatures.]

\section{Transport en couche d'inversion.}

Cette partie présente le formalisme du transport nécessaire au calcul des différents coefficients de transport tels que conductivité, mobilité de Hall ou de dérive, facteur de Hall, etc... dans le système électronique à $2 \mathrm{D}$ d'une couche d'inversion. A cet effet nous utilisons le formalisme de Kubo-Greenwood [5] très employé dans l'étude des matériaux désordonnés, dont l'unité de forme permet d'obtenir les paramètres 
de transport du régime métallique (dégénéré) au régime semiconducteur (non dégénéré). Les mécanismes de collision des porteurs les plus significatifs pour les couches d'inversion seront également décrits. Enfin nous examinerons l'influence des effets de localisation sur les propriétés de transport.

3.1 CAS D'UNe SEULe SOUS-BANDE. - De manière générale la conductivité électrique macroscopique d'un système électronique s'obtient à partir de la conductivité énergétique $\sigma(E)$ par l'intégrale de KuboGreenwood [5] :

$$
\sigma(T)=\int_{E_{\mathrm{c}}}^{+\infty} \sigma(E)\left(-\frac{\delta f}{\delta E}\right) \mathrm{d} E
$$

où $f$ est la fonction de Fermi-Dirac; $f=1 /(1+$ $\left.\exp \left(E_{\mathrm{c}}-E_{\mathrm{F}}\right) / k T\right)$ et $E_{\mathrm{c}}$ dénote le seuil de mobilité qui en présence de désordre ne coïncide pas avec le bord de bande $E_{0}$. Dans le cas d'un système à 2D et pour le transport au-dessus du seuil de mobilité on a $[5,9,10]$ :

$$
\sigma(E)=q\left(E-E_{\mathrm{c}}\right) \mu(E) N(E)
$$

où $\mu(E)$ est la mobilité énergétique fonction de l'énergie cinétique $\left(E-E_{\mathrm{c}}\right)$ par le biais des lois de diffusion (cf. § 3-3) et $N(E)$ la densité d'états à 2D.

$\sigma(E)$ étant donnée, la conductivité de la couche d'inversion (Eq. (8)), dépend de façon complexe de la température $T$ et de la concentration des porteurs $N_{\mathrm{s}}$ sachant que le niveau de Fermi est déterminé par la relation (2). Il est important de noter que, dans le cas où le seuil de mobilité $E_{\mathrm{c}}$ est au-dessus du bord de bande, les électrons d'énergie inférieure à $E_{\mathrm{c}}$, sont localisés et ont une contribution négligeable à la conductivité par rapport à celle due aux électrons délocalisés d'énergie supérieure à $E_{\mathrm{c}}$. Néanmoins, les électrons localisés peuvent participer au transport du courant selon un mécanisme de conduction par saut (" variable range hopping ") notable qu'à très basse température $(T<10-20 \mathrm{~K})[1,5]$.

En remarquant que dans la relation (8), chaque tranche d'énergie contribue en parallèle au transport, la mobilité de Hall à faible champ magnétique (excluant tout effet quantique dû au champ magnétique) s'obtient après généralisation d'une analyse bicouche [11] sous la forme :

$$
\mu_{\mathrm{H}}=\frac{1}{\sigma} \int_{E_{\mathrm{c}}}^{+\infty} \mu(E) \sigma(E)\left(-\frac{\delta f}{\delta E}\right) \mathrm{d} E .
$$

La mobilité de dérive $\mu_{\mathrm{D}}$ est définie par la relation usuelle :

$$
\mu_{\mathrm{D}}=\frac{\sigma}{q N_{\mathrm{s}}}
$$

où $N_{\mathrm{s}}$ est la concentration totale d'électrons dans la couche d'inversion, $\sigma$ la conductivité donnée par la relation (8). Il est clair que $\mu_{\mathrm{D}}$ est une mobilité effective en général différente de la mobilité de Hall. En effet, lorsque le seuil de mobilité est au-dessus du bord de bande, le nombre de porteurs effectivement libres est inférieur au nombre total d'électrons dans la bande. De sorte que, d'après la relation (11), la mobilité effective devient inférieure à la mobilité de dérive réelle des porteurs au-dessus du seuil de mobilité.. En absence d'états localisés en bord de bande $\left(E_{\mathrm{c}}\right.$ confondu avec $E_{0}$ ) et d'anisotropie de la structure de bande à 2D, le facteur de Hall $r=\mu_{\mathrm{H}} / \mu_{\mathrm{D}}$, qui est en général supérieur à 1 , prend la valeur 1 en régime métallique (statistique dégénérée) [12].

3.2 CAS DE PLUSIEURS SOUS-BANDES. - Les coefficients de transport d'une couche d'inversion comportant plusieurs sous-bandes actives proviennent de la contribution en parallèle de chaque sous-bande au transport du courant (les mécanismes de collision inter-sousbandes peuvent être négligés pour des températures inférieures à l'ambiante et à faible champ électrique de dérive $[1,13])$.

La conductivité globale $\sigma(T)$ s'écrit donc simplement [1] :

$$
\sigma(T)=\sum_{i} \sigma_{i}(T)
$$

où $\sigma_{i}(T)$ est la conductivité de la ième sous-bande (donnée par la relation (8) dans laquelle on prend les caractéristiques énergétiques de la sous-bande considérée).

La mobilité de Hall $\mu_{\mathrm{H}}$ devient :

$$
\mu_{\mathrm{H}}=\frac{\sum_{i} \mu_{\mathrm{H}_{i}} \sigma_{i}}{\sigma}
$$

où $\mu_{\mathrm{H}_{i}}$ est la mobilité de Hall de la $i$-ème sous-bande (donnée par la Rel. (10)).

La mobilité de dérive $\mu_{\mathrm{D}}$ s'obtient de manière analogue au moyen de la relation(11).

3.3 PrincipauX mÉCANISMES DE COLlisions. - De façon générale, comme dans les semiconducteurs à 3 dimensions, on peut discuter les lois de dispersion énergétique de la mobilité $\mu(E)$ en terme de probabilité de collision $1 / \tau(E)$ conformément à la relation : $\mu(E)=q \tau(E) / m^{*}$. La probabilité de collision globale résulte de la contribution en série de différents mécanismes de collision de probabilité $1 / \tau_{k}(E)$ et, d'après la loi de Mathiessen [9], est égale à la somme de chacune des probabilités $\left(1 / \tau(E)=\sum_{k} 1 / \tau_{k}(E)\right)$. En ce qui concerne les couches d'inversion, les principaux mécanismes de collision des porteurs sont les suivants [1] :

- collisions coulombiennes,

- collisions sur la rugosité de surface,

- collisions sur les phonons. 
En général seuls les deux premiers mécanismes sont prédominants en dessous de la température ambiante; les collisions sur les phonons n'interviennent qu'à haute température ou sont « masquées » par les autres mécanismes.

Pour notre part, nous adopterons une description synthétique mais simplifiée des lois de collisions alliant la diffusion coulombienne et la rugosité de surface, discutée par Stern [14], dans laquelle, à basse énergie $\mu(E)$ varie en $E$ alors qu'à haute énergie $\mu(E)$ varie en $E^{-\alpha}$ avec $\alpha \# 1-1,5$. On peut alors formuler la mobilité énergétique sous une forme condensée :

$$
\mu(E)=2 \mu_{\mathrm{M}}\left[\left(\frac{E}{E_{\mathrm{M}}}\right)^{\alpha}+\left(\frac{E_{\mathrm{M}}}{E}\right)\right]^{-1} .
$$

Dans ce cas $\mu_{\mathrm{M}}$, valeur sensiblement égale à la valeur maximale de la mobilité, dépend de l'amplitude des collisions (inversement proportionnel à la densité de centres coulombiens et/ou aux dimensions caractéristiques de la rugosité de surface) et $E_{\mathrm{M}}$, valeur sensiblement égale à la valeur de l'énergie pour laquelle la mobilité est maximale, dépend de l'importance relative des deux mécanismes considérés. Nous verrons par la suite que, bien que simple, cette formulation de la mobilité énergétique sera suffisante pour expliquer dans leur globalité les propriétés de transport dans une couche d'inversion.

Il est à noter que Lee et al. [15] ont récemment proposé une loi de $\mu(E)$ analogue à celle de la relation (14) en invoquant un mécanisme de collisions sur les impuretés ionisées tenant compte de l'écrantage du potentiel du centre diffuseur. Dans ce cas $\mu_{M}$ dépend de l'inverse du nombre de centre ionisés (comme dans l'analyse de Stern [14]) mais aussi de l'inverse de la longueur d'écran $\lambda_{2 \mathrm{D}}$ (cf. 2.3); $E_{\mathrm{M}}$ est inversement proportionnel à la longueur d'écran et à l'extension spatiale de la sous-bande (perpendiculairement à l'interface). Ce dernier point montre que, même si la loi de $\mu(E)$ (Eq. (14)) est acceptable, son origine exacte reste encore controversée.

3.4 Localisation DES PORTEURS. - Les effets de localisation des porteurs en bord de bande sont maintenant bien connus. En particulier, l'étude du transport à l'interface $\mathrm{Si}_{-} \mathrm{SiO}_{2}$ a été déterminante pour la mise en évidence de la transition de Mott-Anderson, caractéristique de la présence de queue de bande d'états localisés en bord de bande [5]. D'une façon générale, la formation d'états localisés en bord de bande est due au désordre attribué dans le cas de l'interface oxyde semiconducteur à des fluctuations du potentiel de surface $[1,5,16]$. Ces fluctuations de potentiel dont l'écart type de la distribution, déduit de mesures de conductance dynamique sur des capacités MOS, peut atteindre quelques dizaines de meV [17], sont responsables d'une dégradation notable de la mobilité effective (de dérive) de T.MOS [18].

En adoptant la même démarche que dans les matériaux à 3D [19], on peut caractériser dans l'ap- proximation d'une densité d'états rectangulaire [20], - le taux de désordre introduit par les fluctuations de potentiel, par la largeur énergétique, notée $\Gamma(\Gamma=$ $\left.E_{\mathrm{c}}-E_{0}\right)$, de la plage d'états localisés située en bord de bande. Une approche plus rigoureuse de cette question peut être menée à bien en considérant une distribution gaussienne du potentiel [19, 21], néanmoins pour des raisons de simplicité de calcul, nous nous limiterons ici à l'approximation de la densité d'états rectangulaire. Ainsi, de même qu'en 3D [19], $\Gamma$ dépend de l'écart type $\sigma_{\mathrm{s}}$ de la distribution du potentiel de surface; en première approximation on considère que $\Gamma \# \sigma_{\mathbf{s}}$.
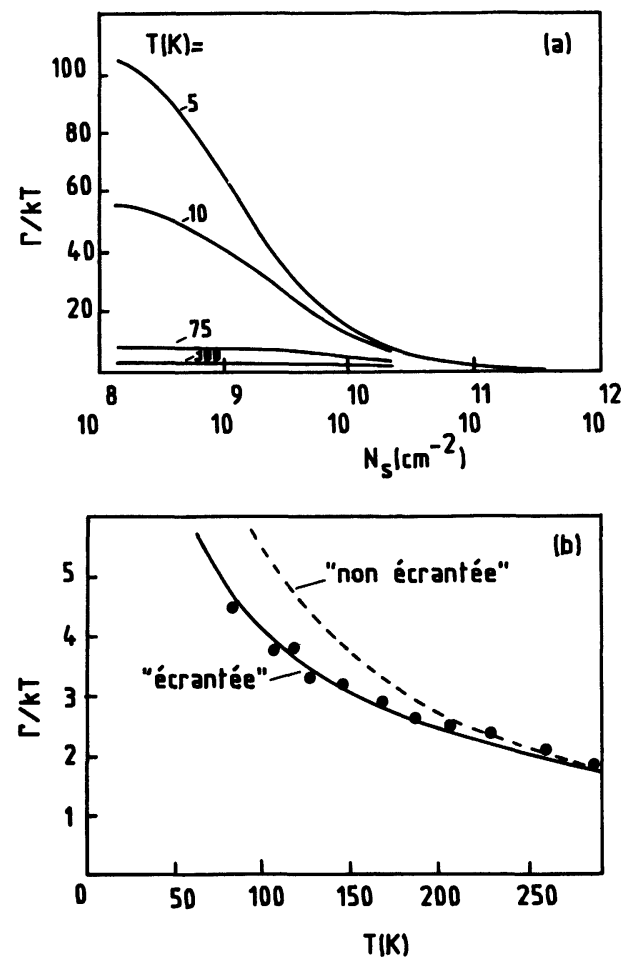

Fig. 3. - (a) Variations du taux relatif de désordre $\Gamma / k T$ avec la concentration de porteurs $N_{\mathrm{s}}$ à différentes températures. (b) Comparaison des variations expérimentales (d'après Nicollian [22]; $\mathrm{Si}(100)$, épaisseur d'oxyde $800 \AA \AA$ ) et théoriques (traits plein et pointillé) du taux relatif de désordre $\Gamma / k T$ avec la température $T\left(N_{\mathrm{s}} \# 2 \times 10^{9} \mathrm{~cm}^{-2}\right)$; en trait plein version écrantée (Rel. (16)), en trait pointillé version nonécrantée $(\Gamma=\mathrm{Cte})$.

[(a) Variations of the relative rate of disorder $\Gamma / k T$ with carrier concentration $N_{\mathrm{s}}$ for different temperatures. (b) Comparison between experimental (after Nicollian [22]; Si(100), oxyde thickness $800 \AA$ ) and theoretical (solid and dashed lines) variations of the relative rate of disorder $\Gamma / k T$ with temperature $T\left(N_{\mathrm{s}} \# 2 \times 10^{9} \mathrm{~cm}^{-2}\right)$; in full line screened version (Rel. (16)), in dashed line non-screened version $(\Gamma=\mathrm{Cte})$.]

Dans le cas où la distribution spatiale des fluctuations de potentiel est de longue ou moyenne portée, la théorie de Brews [18] permet de relier $\sigma_{\mathrm{s}}$ à l'écart type $\sigma_{\mathrm{q}}$ des fluctuations de charges dans l'isolant de 
grille selon la relation (en négligeant les états d'interface) :

$$
\sigma_{\mathrm{s}} \# \frac{\sigma_{\mathrm{q}}}{C_{\mathrm{ox}}+C_{\mathrm{D}}+C_{i}}
$$

où $C_{\text {ox }}$ est la capacité de l'isolant de grille, $C_{\mathrm{D}}$ la capacité relative à la charge déplétée et $C_{i}$ la capacité relative à la charge d'inversion.

La relation (15) peut être réécrite en fonction des longueurs caractéristiques associées aux capacités sous la forme :

$$
\sigma_{\mathrm{s}}=\frac{\sigma_{\mathrm{q}}}{\varepsilon / l_{\mathrm{ox}}+\varepsilon / l_{\mathrm{D}}+\varepsilon / \lambda_{2 \mathrm{D}}}
$$

où $l_{\mathrm{ox}}$ est l'épaisseur de l'isolant de grille, $l_{\mathrm{D}}$ l'épaisseur moyenne de la région désertée et $\lambda_{2 \mathrm{D}}$ la longueur d'écran associée à la couche d'inversion (cf. § 2-3).

Pour des dopages de substrat usuels de $10^{15}$ à $10^{16}$ impuretés $/ \mathrm{cm}^{3}$, l'épaisseur de la région désertée vaut typiquement 1000 à $3000 \AA$. Elle est donc très supérieure aux épaisseurs de l'isolant de grille des MOS de la VLSI (typiquement $l_{\text {ox }}<500 \AA$ ) et sa contribution dans la relation (16) pourra être négligée. De plus, on voit sur la figure 2 que pour des concentrations $N_{\mathrm{s}}$ faibles, la longueur d'écran est supérieure à $l_{o x}$, de sorte que l'influence des fluctuations de potentiel est maximale :

$$
\sigma_{\mathrm{s} \max } \# \sigma_{\mathrm{q}} \frac{l_{\mathrm{ox}}}{\varepsilon} \text {. }
$$

En revanche, pour des concentrations $N_{\mathrm{s}}$ fortes, on a $\lambda_{2 \mathrm{D}} \ll l_{\text {ox }}$, ce qui traduit un écrantage important; il vient pour l'écart type :

$$
\sigma_{\mathrm{s}} \# \sigma_{\mathrm{q}} \frac{\lambda_{2 \mathrm{D}}}{\varepsilon} \text { soit encore } \sigma_{\mathrm{s}} \# \sigma_{\mathrm{s} \max }\left(\frac{\lambda_{2 \mathrm{D}}}{l_{\mathrm{ox}}}\right) \text {. }
$$

Il est clair d'après les relations (17) et (18) que le taux de désordre $\Gamma\left(\# \sigma_{\mathrm{s}}\right)$, partant le nombre d'états localisés en bord de bande, est très fortement dépendant via la longueur d'écran, de la concentration totale de porteurs dans la couche d'inversion et de la température. Ceci aura une grande répercussion sur la valeur de la mobilité effective d'une couche d'inversion à forte localisation. La figure $3 a$ donne une illustration synthétique des variations du taux relatifs de désordre $(\Gamma / k T)$ avec la concentration de porteurs pour différentes températures (Rel. (16)). La figure $3 \mathrm{~b}$ montre de même les variations du taux de fluctuation relatif en fonction de la température pour des concentrations faible $\left(N_{\mathrm{s}} \# 10^{9} / \mathrm{cm}^{2}\right)$. On note que l'effet d'écran apparaît comme un élément déterminant pour rendre compte de la dépendance expérimentale de $\Gamma / k T$ avec la température [22]. En particulier, la diminution à basse température de la longueur d'écran $\lambda_{2 D}$ (cf. § 3.3) est responsable d'une réduction du taux relatif de fluctuation $\Gamma / k T$ par rapport à ce que l'on aurait en négligeant l'effet d'écran (cf. Fig. 3b).

\section{Résultats et discussions.}

Dans cette partie nous comparons les résultats issus du modèle de transport électronique décrits précédemment aux résultats expérimentaux typiques obtenus sur des couches d'inversion de T.MOS.

4.1 INFLUENCE DES COLLISIONS. - L'influence des mécanismes de collision sur la conductivité et la mobilité, se manifeste par l'intermédiaire de la loi de dispersion de la mobilité énergétique $\mu(E)$ via les relations de transport (8) et (10). Deux procédures expérimentales sont en général utilisées pour mettre en évidence les mécanismes de collision : soit, on mesure la mobilité de Hall en fonction de la concentration de porteurs (c'est-à-dire en fonction de la tension de grille) à température fixée; soit, on mesure la mobilité de Hall en fonction de la température en gardant constante la concentration de porteurs. Il est clair que, en réalité, ces deux méthodes sont
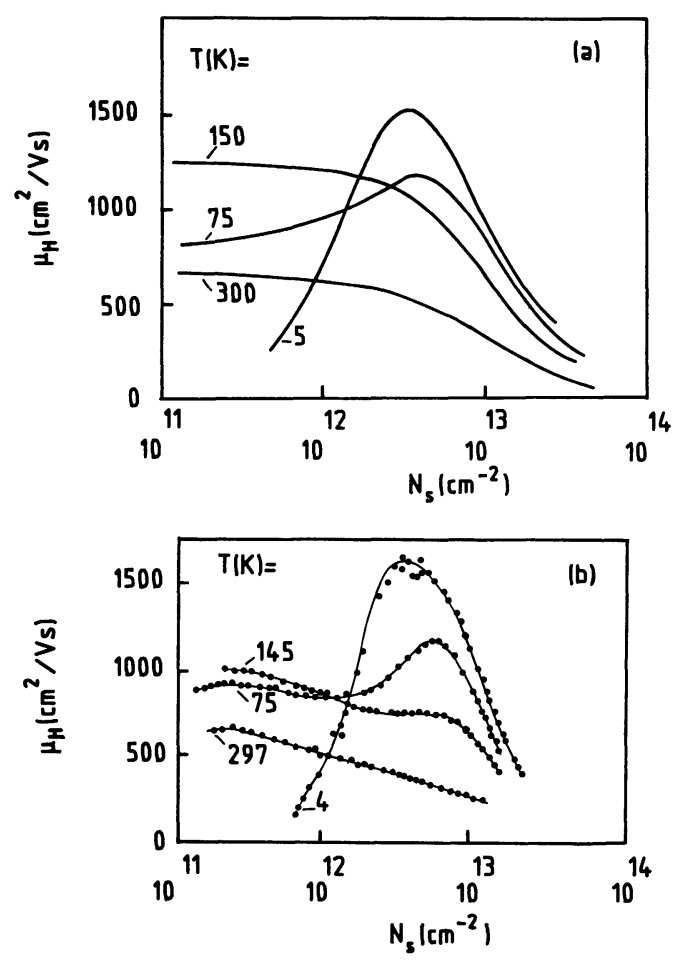

Fig. 4. - (a) Variations théoriques de la mobilité de Hall avec la concentration de porteurs dans une couche d'inversion pour différentes températures (paramètres utilisés : $\mu_{\mathrm{M}}=1600 \mathrm{~cm}^{2} / \mathrm{Vs}, E_{\mathrm{M}}=15 \mathrm{meV}, \alpha=1$; cf. $\S 3.3$ ). (b) Variations expérimentales typiques de la mobilité de Hall avec la concentration de porteurs dans une couche d'inversion pour différentes températures (d'après Fang et Fowler [23]; T.MOS/Si(100), canal N).

[(a) Theoretical variations of the Hall mobility with carrier concentration for an inversion layer at different temperatures (used parameters : $\mu_{\mathrm{M}}=1600 \mathrm{~cm}^{2} / \mathrm{Vs}, E_{\mathrm{M}}=15 \mathrm{meV}$, $\alpha=1$; cf. $\S 3.3$ ). (b) Typical experimental variations of the Hall mobility with carrier concentration for an inversion layer at different temperatures (after Fang and Fowler [23]; T.MOS/Si(100), N channel).] 
équivalentes. Néanmoins, elles permettent de dissocier plus facilement l'effet de l'un ou l'autre des paramètres considérés sur les coefficients de transport.

La figure $4 \mathrm{a}$ montre les variations de la mobilité de Hall avec la concentration de porteurs $N_{\mathrm{s}}$, obtenues à l'aide du modèle décrit ci-dessus (cf. § 3, Eqs. (8), (10) et (14)) dans l'hypothèse d'une couche d'inversion n'ayant qu'une sous-bande active. La figure $4 \mathrm{~b}$ donne une illustration des variations expérimentales typiques de $\mu_{\mathrm{H}}$ en fonction de $N_{\mathrm{s}}$. Le comportement global de la mobilité $\mu_{\mathrm{H}}$ en fonction de $N_{\mathrm{s}}$ est donc reproduit de façon satisfaisante sur une large plage de concentration et de température grâce au modèle utilisé. En particulier, il est important de noter que la diminution de la mobilité à forte charge d'inversion apparaît comme une conséquence directe de la dégénérescence de la couche d'inversion, même à température ambiante (conduction en régime métallique) et non, comme il est souvent dit, à un effet du fort champ électrique de grille. A basse température, la couche d'inversion reste pratiquement dégénérée sur toute la plage de concentration balayée, de sorte que la courbe en cloche obtenue pour la mobilité, est représentative de la mobilité énergétique $\mu(E)$ (donc des lois énergétiques de diffusion) car, en régime de conduction métallique, la valeur de $\mu_{\mathrm{H}}$, d'après la relation (10), s'identifie à la valeur que prend $\mu(E)$ au niveau de Fermi : $\mu_{\mathrm{H}} \# \mu\left(E_{\mathrm{F}}\right)$.

De même, la figure 5 montre les variations théoriques et expérimentales de la mobilité en fonction de la température entre 5 et $300 \mathrm{~K}$ pour différentes concentrations de porteurs. Comme précédemment, le comportement global de la mobilité est simulé correctement sur la plage de température considérée. La disparition avec l'accroissement de la concentration de porteurs de la courbe en cloche observée à faible concentration, caractéristique de la présence de deux mécanismes de collision en régime semiconducteur, est également reproduit.

$\mathrm{Si}$ dans leur ensemble, les courbes expérimentales $\mu_{\mathrm{H}}(T)$ et $\mu_{\mathrm{H}}\left(N_{\mathrm{s}}\right)$ sont bien décrites par le modèle considéré ici, en revanche, le comportement détaillé de la mobilité de Hall n'est pas reproduit avec exactitude. Les deux raisons majeures qui justifie ces écarts sont, d'une part le fait de ne prendre en compte qu'une seule sous-bande (cela est sensible notamment à faible concentration et/ou à faible température) et d'autre part, la mauvaise connaissance des lois précises des mécanismes de collision.

Sur la figure 6 nous montrons, à titre indicatif, les variations du facteur de Hall $r$ (cf. $\S 3.1$ ) avec la concentration de porteurs pour différentes températures. Il est important de souligner que, si pour des températures proches de l'ambiante, on peut confondre à $20 \%$ près la mobilité de Hall et la mobilité de dérive, en revanche, à très basse température $(T \# 5 \mathrm{~K})$ et pour des concentrations faibles (régime de conduction semiconducteur) l'écart entre la mobilité de Hall et la mobilité de dérive devient non négligeable.
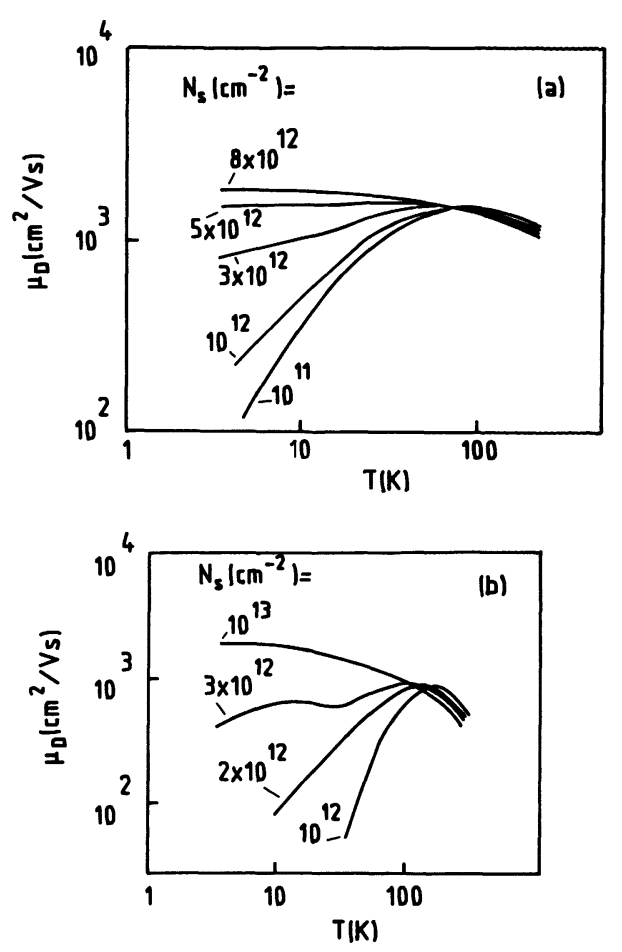

Fig. 5. - (a) Variations théoriques de la mobilité avec la température pour différentes concentrations de porteurs (paramètres : id. Fig. 4). (b) Variations expérimentales de la température pour différentes concentrations de porteurs (d'après Ref. [23]; T.MOS/Si(100), canal N).

[(a) Theoretical variations of the mobility with temperature at different carrier concentrations (parameters : id. Fig. 4). (b) Experimental variations of the mobility with temperature at different carrier concentrations (after Ref. [23]; T.MOS/ Si(100), N channel).]

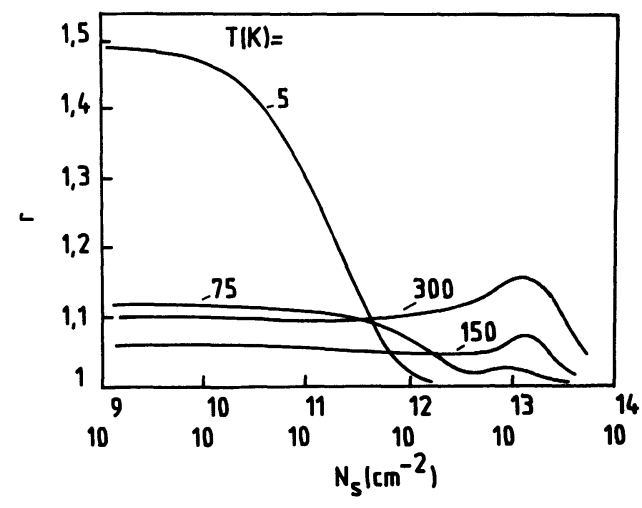

Fig. 6. - Variations théoriques du facteur de Hall avec la concentration de porteur pour différentes températures.

[Theoretical variations of the Hall factor with carrier concentration at different temperatures.]

4.2 INFLUENCE DU DÉSORDRE. - L'influence du désordre sur la conductivité ou la mobilité de dérive est en général étudiée en analysant, soit la dépendance logarithmique de la conductivité (ou de la mobilité 
de dérive) avec l'inverse de la température (régime activé), soit par la dépendance de la mobilité de dérive avec la concentration de porteurs en régime de faible inversion.

La première procédure a fait l'objet de nombreuses discussions dans le cadre de l'étude de la transition de Mott-Anderson [5, 24]. Elle a permis entre autre de discuter de l'existence du concept de conductivité métallique minimale, en analysant la dépendance de la valeur extrapolée de la conductivité à haute température à partir du régime activé efficient à basse température [20,24].

La figure 7 donne une illustration des variations de la mobilité de dérive $\mu_{\mathrm{D}}$ en fonction de l'inverse de la température caractéristique du régime activé observé à basse température dans une couche d'inversion. L'existence d'une région activée est significative de la présence d'états localisés en bord de bande, partant d'un seuil de mobilité. En effet, à basse température et

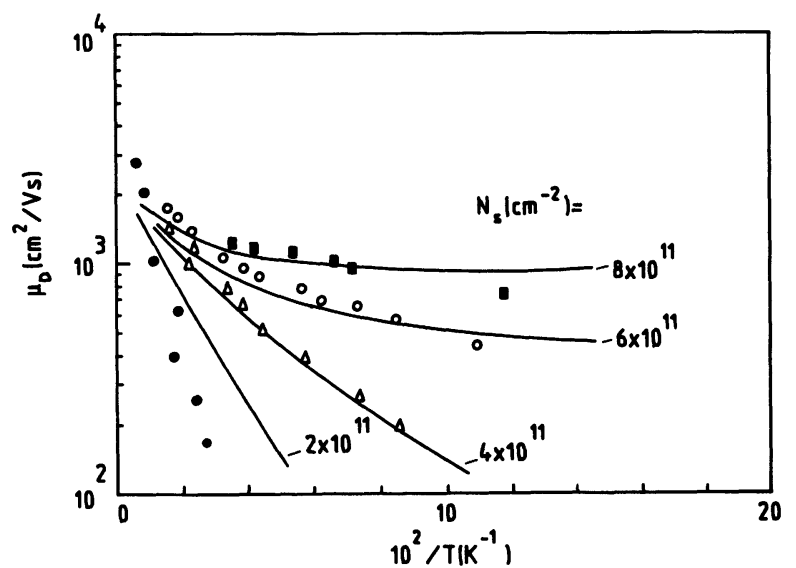

Fig. 7. - Variations théoriques (courbes continues) et expérimentales (d'après Réf. [23]; T.MOS/Si(100), canal N) du logarithme de la mobilité de dérive avec l'inverse de la température pour différentes concentrations de porteurs (paramètres de désordre : $\Gamma_{\max }=6 \mathrm{meV}$, cf. § 3.4).

[Theoretical (solid curves) and experimental (after Ref. [23]; T.MOS/Si(100), N channel) variations of the drift mobility with reverse temperature for different carrier concentration (disorder parameter : $\Gamma_{\max }=6 \mathrm{meV}$, cf. § 3.4).]

pour des concentrations pas trop élevées, le niveau de Fermi se stabilise au-dessus du bord de bande tout en restant en dessous du seuil de mobilité, de sorte que le nombre de porteurs excités au-dessus de $E_{\mathrm{c}}$ garde un comportement activé avec la température; l'énergie d'activation prenant une valeur $W \# E_{\mathrm{c}}-$ $E_{\mathrm{F}}\left(E_{\mathrm{F}}>E_{0}\right)$. Quand la concentration augmente, le niveau de Fermi vient se placer au-dessus du seuil de mobilité (transition de Mott-Anderson), et donne lieu à un transport de type métallique (absence d'activation) avec une mobilité quasi constante à basse température, exception faite des corrections de faible localisation [1]. L'ensemble du comportement de la conductivité ou de la mobilité de dérive décrit ci-avant est donc justiciable en grande partie du modèle de désordre que nous avons détaillé au paragraphe 3.4. Cela montre l'intérêt que l'on doit porter au rôle du désordre dans l'évaluation des coefficients de transport dans les couches d'inversion surtout pour des températures de fonctionnement basses.

Cependant, même pour des températures voisines de l'ambiante, l'influence du désordre sur la mobilité de dérive n'est pas négligeable. En effet, lorsque la concentration de porteurs est faible (ce qui est le cas en régime d'inversion faible), la couche d'inversion n'est pas dégénérée de sorte que le nombre de porteurs effectivement libres, c'est-à-dire au-dessus du seuil de mobilité, est inférieur au nombre de porteurs total dans la couche d'inversion d'un facteur $\exp (-\Gamma / k T)$ où $\Gamma / k T$ est le taux relatif de désordre (cf. § 3-4).

La figure 8 montre l'évolution de la mobilité de dérive en fonction de la concentration de porteurs entre l'inversion faible et l'inversion forte pour différents taux de désordre. La valeur du taux maximum de désordre $\Gamma_{\max }$ est reliée à l'écart type des fluctuations de charges $\sigma_{\mathrm{q}}$ par la relation (17) (pour une épaisseur d'isolant $l_{\text {ox }}=500 \AA$, on a $\Gamma_{\max }=10 \mathrm{meV}$ quand $\sigma_{\mathrm{q}} \# 1,3 \times 10^{10} \mathrm{q} / \mathrm{cm}^{2}$ ). La diminution de la mobilité observée à faible concentration de porteurs dépend donc de manière critique du taux de désordre $\Gamma$. En particulier, il est important de souligner que la qualité technologique de l'interface caractérisée par le paramètre intensif $\sigma_{q}$, détermine l'intensité du désordre effectif $\Gamma_{\max }$ pour une épaisseur d'isolant donné. Notons enfin qu'à qualité technologique égale $\left(\sigma_{\mathrm{q}}=\right.$

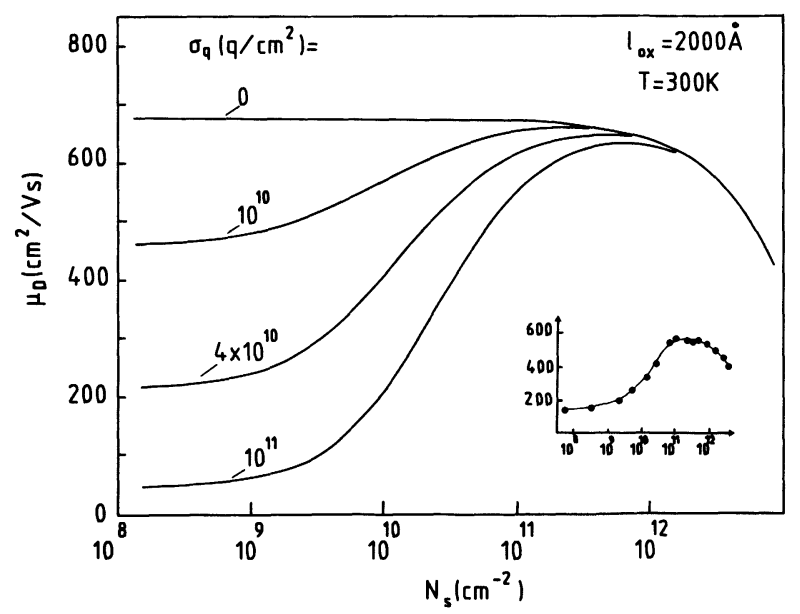

Fig. 8. - Variations de la mobilité de dérive avec la concentration de porteurs pour différents taux de désordre. En insertion, comportement expérimental typique (d'après Chen et Muller [25]; T.MOS/Si(100), canal N).

[Variations of the drift mobility with carrier concentrations for different disorder rates. In insertion, typical experimental results (after Chen and Muller [25]; T.MOS/Si(100), N channel).] 
Cte), le taux de désordre de la couche d'inversion décroît si l'on diminue l'épaisseur de l'isolant de grille, ce qui est le cas en VLSI (cf. Rel. (17)).

\section{Conclusion.}

Nous avons présenté dans cet article un modèle de transport dans la couche d'inversion d'un T.MOS basé sur le formalisme de Kubo-Greenwood. Ce modèle répond pour l'essentiel aux objectifs que nous nous étions fixés en début d'étude. En effet, il prend en compte les principaux phénomènes physiques découlant des conditions actuelles de fonctionnement des T.MOS (fort champs électrique de surface, température basse) à savoir, la quantification de la couche d'inversion, l'emploi de la statistique de Fermi-Dirac, l'effet de la localisation des porteurs.

Nous avons vu que, dans une large mesure, les coefficients de transport dans la couche d'inversion, sont décrits de façon satisfaisante sur une large gamme de température et de concentration de porteurs. Cela est rendu possible grâce à l'utilisation du forma- lisme de transport de Kubo-Greenwood qui autorise une formulation globale du transport valable quelles que soient la température et la concentration de porteurs.

En outre, le comportement global des coefficients de transport en fonction de la température et/ou de la concentration de porteurs, est justiciable en grande partie d'hypothèses simples en ce qui concerne l'influence du désordre et des lois de diffusion des porteurs.

Ce travail qui constitue une première approche de la modélisation du transport dans la couche d'inversion de T.MOS, pourra être approfondi par la suite en tenant compte de la contribution de plusieurs sousbande au transport et de lois plus sophistiquées des mécanismes de diffusion des porteurs.

Il ressort également que la modélisation du fonctionnement des T.MOS de la VLSI (faible épaisseur d'isolant de grille, MOS cryogéniques) ne saurait ignorer que les limites de validité des approximations utilisées dans les modèles actuels de composant, seront atteintes dans un proche avenir, de sorte que la crédibilité de tels modèles, sera fortement mise en cause.

\section{Bibliographie}

[1] Ando,T., Fowler, A. and Stern, F., Rev. Mod. Phys. 54 (1981) 437.

[2] Baccarani, G. and Wordeman, M. R., Proc. of the Int. Electron. Devices Meeting, San Francisco, USA (1982) 278.

[3] Yoshino, H., Proc. of the 13th European Solid State Device Research Conference, Canterbury, UK (1983) vol. F7, 55.

[4] Pals, J. A., Phys. Rev. 7 (1972) 754.

[5] MotT, N. and DAviEs, E., Electronic processes in noncrystalline materials (Clarendon, Oxford) 1979.

[6] Gnadinger, A. and Talley, H., Solid State Electron. 13 (1970) 1301.

[7] Stern, F., CRC Critic. Rev. Solid State Sci. 5 (1974) 499.

[8] STERN, F., Surf. Sci. 73 (1978) 197.

[9] Ziman, J., Electrons and Phonons (Clarendon, Oxford) 1960.

[10] Dugdale, J., The electrical properties of metals and alloys (Arnold, London) 1977.

[11] Ghibaudo, G. et Kamarinos, G., Rev. Phys. Appl. 17 (1982) 133.
[12] Sakaki, H. and Sugano, T., Japan J. Appl. Phys. 10 (1971) 1016.

[13] Ezawa, H., Surf. Sci. 58 (1976) 25

[14] Stern, F., Phys. Rev. Lett. 44 (1980) 1469.

[15] Lee, J., Spector, H. and Arora, V., Appl. Phys. Lett. 42 (1983) 362.

[16] Brews, J. R., J. Appl. Phys. 46 (1975) 2181.

[17] Nicollian, E. H. and Brews, J. R., MOS Physics and Technology (Wiley, New York) 1982.

[18] Brews, J. R., J. Appl. Phys. 46 (1975) 2193

[19] Robert, J. L., Pistoulet, B., Raymond, A., Dusseau, J. M. and Martin, G. M., J. Appl. Phys. 50 (1979) 349.

[20] Ghibaudo, G., J. Phys. C 17 (1984) 3067.

[21] Arnold, E., Appl. Phys. Lett. 25 (1974) 706.

[22] Nicollian, E. H., CRC Critic. Rev. Solid State Sci. 7 (1976) 1.

[23] Fang, F. F. and Fowler, A. B., Phys. Rev. 3 (1968) 619.

[24] Adkins, C. J., J. Phys. C 11 (1978) 851.

[25] Chen, J. T. and Muller, R. S., J. Appl. Phys. 45 (1974) 828. 